\title{
Knots and Random Walks in Vibrated Granular Chains
}

\author{
E. Ben-Naim ${ }^{1,2}$, Z. A. Daya ${ }^{2,3}$, P. Vorobieff ${ }^{2,4}$, and R. E. Ecke ${ }^{2,3}$ \\ ${ }^{1}$ Theoretical Division, ${ }^{2}$ Center for Nonlinear Studies, ${ }^{3}$ Condensed Matter 8 Thermal Physics Group \\ Los Alamos National Laboratory, Los Alamos, NM 87545 \\ ${ }^{4}$ Department of Mechanical Engineering, University of New Mexico, Albuquerque NM 87131
}

\begin{abstract}
We study experimentally statistical properties of the opening times of knots in vertically vibrated granular chains. Our measurements are in good qualitative and quantitative agreement with a theoretical model involving three random walks interacting via hard core exclusion in one spatial dimension. In particular, the knot survival probability follows a universal scaling function which is independent of the chain length, with a corresponding diffusive characteristic time scale. Both the large-exit-time and the small-exit-time tails of the distribution are suppressed exponentially, and the corresponding decay coefficients are in excellent agreement with the theoretical values.
\end{abstract}

PACS: 05.40.-a, 81.05.Rm, 83.10Nn

Topological constraints such as knots [1] and entanglements strongly affect the dynamics of filamentary objects including polymers [2 [5] and DNA molecules [6,7]. Typically, large time scales are associated with the relaxation of such constraints 88.9]. Understanding the physical mechanisms governing the relaxation of such constraints is crucial to characterizing flow, deformation, as well as structural properties of materials consisting of ensembles of macromolecules, e.g., polymers, gels, and rubber.

Scaling techniques, such as de Gennes-Edwards reptation theory, provide a powerful tool for modeling dynamics of topological constraints [8,9]. These are successful when the precise details of the interparticle interactions are secondary relative to the geometric effects. However, topological constraints are difficult to control experimentally and typically, they can be probed only using indirect methods. Here, we introduce a physical system where these difficulties are greatly reduced, thereby enabling a detailed quantitative comparison with theory.
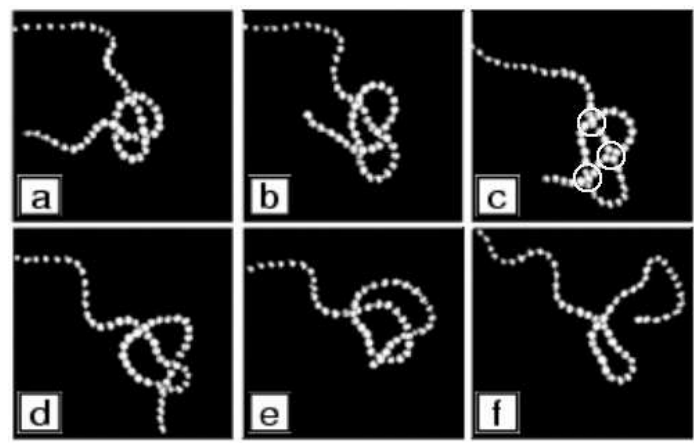

FIG. 1. Illustrative snapshots of the vibrated knot experiment, taken every ten plate oscillation cycles.

In this Letter, we study dynamics of knots in vibrated granular chains. This system has an appealing simplicity as the "molecular weight" of the chain and the driving conditions can be well controlled. Additionally, the topological constraints can be directly observed. We restrict our attention to simple knots and investigate the time it takes for a knot to open. We find that the average unknotting time $\tau$ is consistent with a diffusive behavior $\tau \sim N^{2}$ where $N$ is the number of beads in the chain. We also show that statistical properties of opening times are well described by a one dimensional model where three random walks, representing the three exclusion points governing the knot, interact via excluded volume interactions. This model provides an excellent approximation to the knot survival probability. Furthermore, quantitative predictions of this model including fluctuations in the exit times, as well as the coefficients governing the exponential decay of the extremal tails of the distribution are in excellent agreement with the measured values.

In the experiments, a simple knot was tightly tied in the center of a ball chain and placed onto a vibrating plate. In Fig. 1, we show images representative of the unknotting process starting from a tightly knotted chain, Fig. 1a, through intermediate states, Figs. 1b-e to an unknotted state, Fig. 1f. The chain consists of $N$ hollow nickel-plated steel spheres of diameter $2 R_{\text {bead }}=$ $2.37 \pm 0.02 \mathrm{~mm}$ connected by thin rods of diameter $0.52 \pm 0.02 \mathrm{~mm}$. The maximum extension between two beads is $0.94 \pm 0.01 \mathrm{~mm}$, or roughly $0.8 R_{\text {bead }}$. The stainless steel plate has a diameter $13.40 \mathrm{~cm}$. The beads essentially interact via hard core repulsion (deformation experienced during collisions $<1 \%$ ), and the connecting rods act as nonlinear springs. This bead-spring chain can be viewed as a "granular polymer". Beads experience dissipative inelastic collisions with the plate as well as with other beads, and motion is sustained by injecting energy via the harmonically oscillating plate [10].

Similar to experiments of vertically-vibrated granular materials [11, 12], given the plate height $z=A \sin \omega t$, with amplitude $A$, angular velocity $\omega=2 \pi \nu$, and frequency $\nu$, the driving conditions are characterized by the frequency $\nu$ and the dimensionless acceleration $\Gamma=$ $A \omega^{2} / g$ ( $g$ is the gravitational acceleration). We examined the parameter range $12 \mathrm{~Hz}<\nu<16 \mathrm{~Hz}$ and $1.7<\Gamma<3$, but as statistical properties of the unknotting time were independent of the driving conditions, we report results obtained with $\nu=13 \mathrm{~Hz}$ and $\Gamma=2.40 \pm 0.05$ (variations in the acceleration across the plate are smaller than $2 \%$ ). 


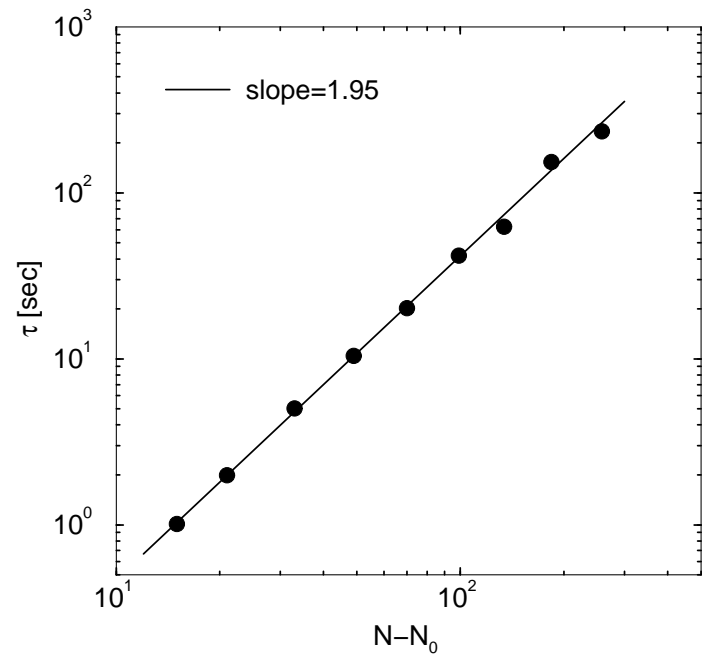

FIG. 2. The average unknotting time $\tau$ versus the chain length $N-N_{0}$. Each data point represents an average over 400 measurements. The line represents a power-law best fit.

Let us first discuss the dependence of the average unknotting time $\tau$ on the chain length $N$. We have measured this time for chains of lengths $30 \leq N \leq 273$. The lower limit was dictated by the knot size as the minimal number of beads associated with the knot is $N_{0}=15 \pm 1$. Additionally, the error in measurement is significant for times much smaller than 1 second, and obtaining statistically significant measurements for large opening times becomes prohibitive. Nevertheless, the results shown in Fig. 2 provide evidence of diffusive behavior via the scaling of $\tau$ with $N$ :

$$
\tau \sim\left(N-N_{0}\right)^{\delta},
$$

with $\delta \cong 2.0 \pm 0.1$ (the rationale for subtracting the knot size $N_{0}$ is given below). A power-law best fit of the data yields an exponent of $\delta=1.95 \pm 0.07$, and the larger error bar was obtained by combining possible systematic errors with the statistical uncertainties.

Next, we examine whether $\tau$ is the only time scale underlying the distribution of opening times. Let $S(t, N)$ be the probability that a knot placed on the vibrating plate at time 0 is still "alive" at time $t$. This probability yields $R(t, N)$, the exit time distribution $R(t, N)=-\frac{d}{d t} S(t, N)$, and the average time is given by $\tau=\int d t t R(t, N)$. The measured survival probabilities suggest that rather than depending on two parameters $t$ and $N, S(t, N)$ is characterized by a single scaling variable

$$
S(t, N)=F(z), \quad z=\frac{t}{\tau} .
$$

As shown in Fig. 3, apart from systematic deviations for the two shortest chains (this data is not used in further analysis), a universal scaling function underlies survival probabilities obtained for different chains. This scaling behavior is very useful as it enables us to combine different sets of measurements and therefore evaluate quantitative characteristics of the distribution with smaller statistical uncertainties.

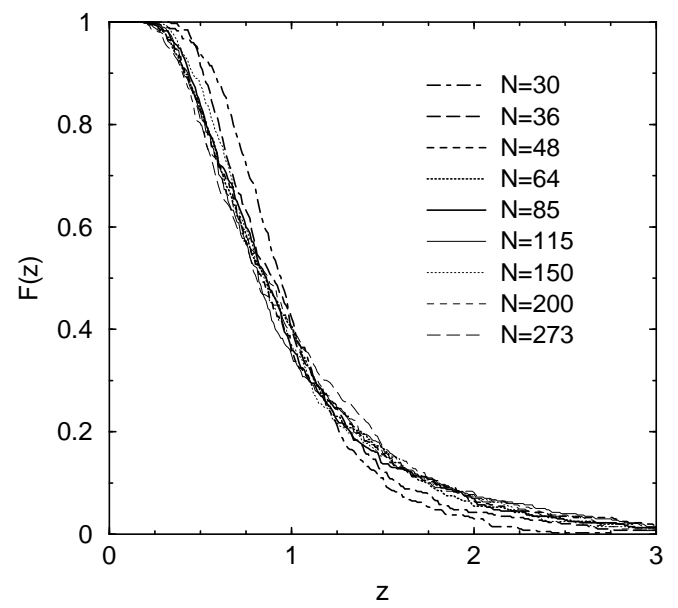

FIG. 3. Scaling of the survival probability. The survival probabilities corresponding to the data points in Fig. 2 are plotted versus the scaling variable $z=t / \tau$. Although $\tau$ changes by two orders of magnitude, the distributions follow a universal scaling curve.

Our theoretical model is based on simple observations of the knot dynamics. Whereas the detailed chain motion is complicated, being determined by wave motion excited in the chain and by collisions between beads and between beads and the plate, the knot moves via a series of short range hops of the cross-links constituting it. When one of the two external cross links defining the knot crosses one of the chain ends for the first time, the knot opens. The diffusive opening times suggest that the hops are random in direction.

In a chain of length $N$ there are $N-1$ links, and as indicated in Fig. 1c, the knot motion appears to be subject to three constraints, namely exclusion points located at positions $1<x_{1}<x_{2}<x_{3}<N-1$. These exclusion points cannot cross each other and, since the knot itself contains $N_{0}$ links, one must have $x_{3}-x_{1}>N_{0}$. Our theoretical model assumes that (i) these points hop randomly, (ii) their motion is uncorrelated, i.e., all three points hop independently, and (iii) the $N_{0}$ links constituting the knot can be divided equally among the three exclusion points, i.e., $x_{i+1}-x_{i}>N_{0} / 3$. With these three simplifying assumptions, we arrive at a model of three identical random walks interacting via excluded volume interactions on a one dimensional lattice. The finite $N_{0} / 3$ size of these three "particles" merely amounts to an overall rescaling of the lattice size $N \rightarrow N-N_{0}$.

Within the model framework, the knot survival probability equals the probability that all three walks remain confined to a finite domain. We are interested primarily in large chains, and hence, we consider the continuum limit $x_{i} \rightarrow \infty, N \rightarrow \infty$ with the variables $x=x_{1} / N, y=x_{2} / N$, and $z=x_{3} / N$ kept finite. Since the three exclusion points perform independent random walks with identical diffusivity $D$, the problem is therefore reduced to diffusion in three dimensions $[13$. Setting the diffusion coefficient to unity by redefining the 
time variable $t \rightarrow D t / N^{2}$, then $P(x, y, z, t)$, the probability that at time $t$ the three walks are at positions $0<x<y<z<1$ respectively evolves according to the diffusion equation $\partial_{t} P=\nabla^{2} P$. The initial conditions read $P(x, y, z, 0)=\delta\left(x-x_{0}\right) \delta\left(y-x_{0}\right) \delta\left(z-x_{0}\right)$ with $x_{0}$ the knot starting position. The reflecting boundary conditions $\left.\left(\partial_{x}-\partial_{y}\right) P\right|_{x=y}=\left.\left(\partial_{y}-\partial_{z}\right) P\right|_{y=z}=0$ ensure that the walks do not cross each other. Finally, the survival probability $S_{3}(t)$, namely the probability that all three walks remain confined to within the box boundary, is obtained by enforcing the absorbing boundary conditions $P(0, y, z, t)=P(x, y, 1, t)=0$, and integrating the probability $S_{3}(t)=\int_{0}^{1} d x \int_{x}^{1} d y \int_{y}^{1} d z P(x, y, z, t)$. The solution $P(x, y, z, t)=3 ! p(x, t) p(y, t) p(z, t)$ can be easily constructed from the solution of the one dimensional problem $\partial_{t} p(x, t)=\partial_{x x} p(x, t)$ subject to the corresponding absorbing boundary conditions $p(0, t)=p(1, t)=0$, and initial conditions $p(x, 0)=\delta\left(x-x_{0}\right)$. Indeed, the product solution satisfies the evolution equation as well as the initial and boundary conditions. Physically, since the walks are identical, the interacting random walk problem can be mapped to a noninteracting problem by simply exchanging the identity of the particles whenever their trajectories cross. In general, $S_{m}(t)$, the survival probability of $m$ random walks is

$$
S_{m}(t)=[s(t)]^{m},
$$

with the single walk survival probability $s(t)=\int_{0}^{1} d x p(x, t)$ obtained by integrating the wellknown solution of the linear diffusion problem 14. $p(x, t)=2 \sum_{n=1}^{\infty} \sin \left(n \pi x_{0}\right) \sin (n \pi x) \exp \left[-(n \pi)^{2} t\right]$,

$$
s(t)=\frac{4}{\pi} \sum_{k=0}^{\infty} \frac{\sin \left[(2 k+1) \pi x_{0}\right]}{2 k+1} e^{-(2 k+1)^{2} \pi^{2} t} .
$$

In the following, we set $x_{0}=1 / 2$.

Statistical properties of the exit time distribution can be determined from the survival probability. For example, $R_{m}(t)$, the exit time probability distribution function is $R_{m}(t)=-\frac{d}{d t} S_{m}(t)$, and moments of the exit time distribution are given by $\left\langle t^{n}\right\rangle_{m}=\int d t t^{n} R_{m}(t)$. As expected, the mean first passage time decreases as the number of walks increases $\tau_{m} \equiv\langle t\rangle_{m}=$ $1 / 8,0.073671,0.056213$ for $m=1,2,3$, respectively. Rescaling the mean exit times to unity via the scaling function $F_{m}(z)=S_{m}\left(z\langle t\rangle_{m}\right)$ allows us to compare the results with the experimental data. As seen from Fig. 4, the three-random-walk model is in remarkable agreement with the data: the two distributions agree to within $4 \%$ in the range $z<3\left(F>10^{-2}\right)$. Furthermore, fluctuations in the mean unknotting times, characterized by the width of the normalized unknotting time distribution $\sigma^{2}=\left\langle z^{2}\right\rangle-\langle z\rangle^{2}=\left(\left\langle t^{2}\right\rangle-\langle t\rangle^{2}\right) /\langle t\rangle^{2}$ are within $1.5 \%$ of each other. For the experimental data one has $\sigma=0.62(1)$, whereas the theoretical values are $\sigma=0.81649(\sqrt{2 / 3}), 0.70495,0.63047$ for $m=1,2,3$.

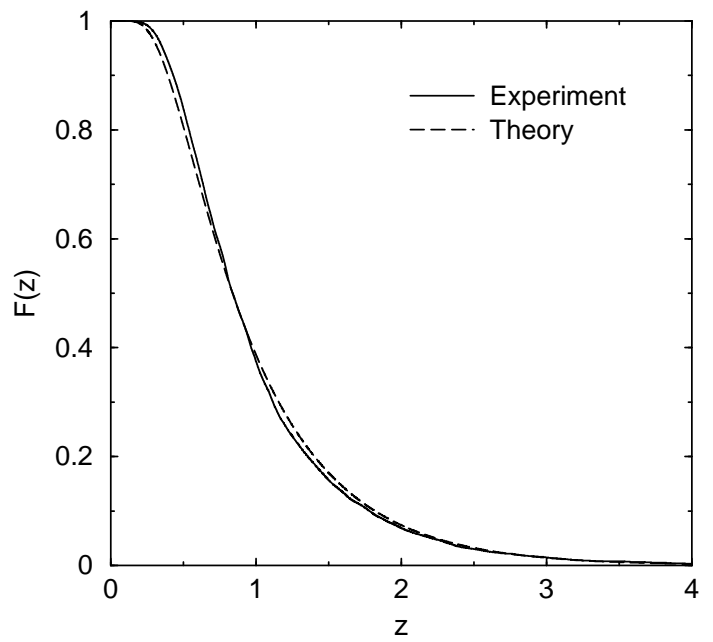

FIG. 4. The survival probability $F(z)$ versus the scaling time variable $z=t / \tau$. The experimental curve represents 6000 data points obtained by aggregating different sets of measurements with 100-400 data points in each set. The theoretical curve is obtained from Eqs. (3)-(4) with $m=3$.

Extremal properties can be studied as well. One may ask "what is the probability that the knot opens in a time equal to $k$ times larger or $1 / k$ smaller than the average time?" (with $k \gg 1$ ). The answer to either questions is "exponentially small". The large tail statistics follow directly from Eq. (4): the first term in the series, corresponding to the largest decay time, governs the long time behavior and $S_{m}(t) \sim \exp \left(-m \pi^{2} t\right)$. The large argument tail of the survival probability is suppressed exponentially

$$
F(z) \sim e^{-\beta z} \quad \text { for } \quad z \gg 1,
$$

with $\beta=m \pi^{2} \tau_{m}$. This exponential behavior is observed experimentally as shown in Fig. 5. The quantitative agreement is striking with the experimental value $\beta=1.65(2)$ within $1 \%$ of the theoretical value corresponding to the three random walk model, $\beta=1.66440$.

In the complementary short time $(t \rightarrow 0)$ limit, the survival probability can be found by performing a steepest descent analysis on the leading large argument $(q \rightarrow \infty)$ behavior of the exit probability Laplace transform obtained by differentiating Eq. (4) $\int d t e^{-q t}\left[-\frac{d}{d t} s(t)\right]=$ $[\cosh (\sqrt{q} / 2)]^{-1}$. In this case one finds that $1-S_{m}(t) \sim$ $\sqrt{t} \exp [-1 / 16 t]$, and consequently, the small argument tail decays exponentially with inverse $z$

$$
1-F(z) \sim \sqrt{z} e^{-\alpha / z} \quad \text { for } \quad z \ll 1,
$$

where $\alpha=1 / 16 \tau_{m}$. Here, the theory suggests an additional algebraic prefactor. Although the data are consistent with such behavior as shown in Fig. 5, we can only address the exponential behavior with our current statistics. The difference in this case is larger: the experimental data yields $\alpha=1.2(1)$ whereas the theoretical value is $\alpha=1.11184$. Nevertheless, it is remarkable that 
even this more subtle statistic is in good agreement with the theoretical predictions.

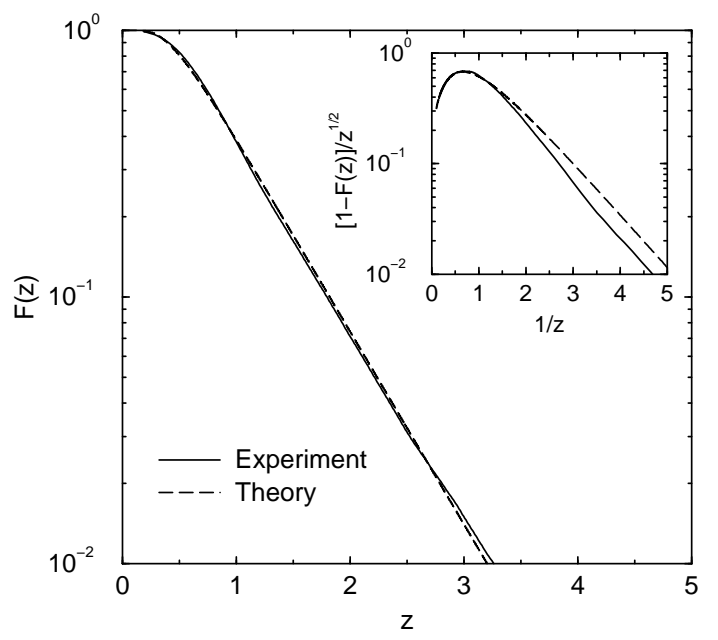

FIG. 5. The exponential tails of the survival probability. The experimental data of Fig. 4 (solid curves) is plotted versus the three random walk theory (dashed curves). The large argument tail is shown by plotting $F(z)$ versus $z$, while the small argument tail is shown in the inset where $[1-F(z)] / \sqrt{z}$ is plotted versus $z^{-1}$.

We also examined other predictions of the theory by placing the knot at off-center starting positions $x_{0} \neq 1 / 2$. One can then study conditional statistics of knots exiting at the near and far ends. Overall, we find that statistical properties such as the overall survival probabilities, the conditional survival probabilities, and the relative fluctuations in the exit times are in reasonable quantitative agreement with the three random walk model. Additionally, we verified that the coefficient characterizing the large tail decay of the distribution is independent of $x_{0}$ in agreement with Eqs. (3)-(4), reflecting that the initial conditions are "forgotten" by long lasting knots.

To test the range of validity of the above results, we studied how $\tau$ and $F(z)$ depend on the driving conditions. Fixing the acceleration at $\Gamma=2.4$, both $\tau$ and $F(z)$ were frequency independent in the range $12 \mathrm{~Hz}<\nu<16 \mathrm{~Hz}$. Setting the frequency at $\nu=13 \mathrm{~Hz}, F(z)$ remained the same in the accessible acceleration range $1.7<\Gamma<3$. This is despite the fact that $\tau$ diverged as the acceleration approached a critical value $\Gamma_{c} \cong 1.6$, below which the cross links did not move and consequently, the knot remained tied. These observations are consistent with recent experiments in vibrated granular layers where the behavior is governed primarily by $\Gamma$ [11,12]. Additionally, by doubling the sidewall diameter, and measuring $\tau, F(z)$, and $\sigma$, we confirmed that the effects of the sidewalls were negligible even for the longest chain. In short, our findings suggest that the driving parameters $(\Gamma, \nu)$ and the chain parameters merely determine the hopping rate $D$, and that $D$ is uniform along the chain and independent of the chain length. In units of the characteristic time scale, $\tau$, opening time statistics are given by a uni- versal scaling function $F(z)$.

The most significant assumption made in our model is that the motion of the exclusion points is uncorrelated. While such correlation is present for small knots, possibly responsible for the larger discrepancy in the short exit time tail and in the survival probability of short chains, it is possible, however, that it becomes negligible beyond some fixed correlation length. In any case, the random walk model can be useful for characterizing isolated topological constraints. Measurements of $\tau(N) \simeq \tau_{3}\left(N-N_{0}\right)^{2} / D$ can be used to extract the constraint size $N_{0}$ as well as the hopping rate $D$. For instance, minimizing the statistical uncertainty in the power law best fit to the data in Fig. 2 yields $N_{0} \cong 15.2$, consistent the actual knot size $N_{0}=15 \pm 1$, while the hopping rate $D=11 \pm 1 \mathrm{sec}^{-1}$ is found to be comparable with the frequency $\nu=13 \mathrm{~Hz}$. Furthermore, the effective number of constraints $m$ can be deduced by comparing the measured $F(z)$ with the theoretical $F_{m}(z)$.

In conclusion, vibrated granular chains provide a useful tool for probing dynamics of topological constraints. As the vibrating plate effectively plays the role of a heat bath, constantly supplying the system with energy, this system may prove useful for studying issues of current interest in polymer dynamics as well as in granular media.

We thank David Egolf and Zoltán Toroczkai for useful discussions. This research is supported by the US DOE (W-7405-ENG-36) and by the Canadian NSERC.

[1] K. Murasugi, Knot Theory and its Applications, (Birkhäuser, Boston, 1996).

[2] K. Koniaris and M. Muthukumar Phys. Rev. Lett. 66, 2211 (1991).

[3] K. Iwata and M. Tanaka, J. Chem Phys. 96, 4100 (1992).

[4] S. R. Quake, Phys. Rev. Lett. 73, 3317 (1994).

[5] E. Ben-Naim, G. S. Grest, T. A. Witten, and A. R. C. Baljon, Phys. Rev. E 53, 1806 (1996).

[6] J. C. Wang, J. Mol Bio 55, 523 (1971).

[7] S. A. Wasserman and N. R. Cozzarelli, Science 232, 951 (1986).

[8] P. G. de Gennes, Scaling Concepts in Polymer Physics (Cornell, Ithaca, 1979).

[9] M. Doi and S. F. Edwards, The Theory of Polymer Dynamics (Clarendon Press, Oxford, 1986).

[10] H. Jaeger and S. Nagel, R. P. Behringer, Rev. Mod. Phys. 68, 1259 (1996).

[11] F. Mello, P. B. Umbanhowar, and H. L. Swinney, Phys. Rev. Lett. 75, 3838 (1995).

[12] J. S. Olafsen and J. S. Urbach, Phys. Rev. Lett. 81, 4369 (1998).

[13] M. E. Fisher, J. Stat Phys. 34, 667 (1984).

[14] H. S. Carslaw and J. C. Yaeger, Conduction of Heat in Solids, (Clarendon Press, Oxford, 1959). 